\title{
Biofeedback Therapy After Sphincter-Preservation Surgery for the Treatment of Rectal Cancer
}

\author{
Ik Yong Kim \\ Department of Surgery, Yonsei University Wonju College of Medicine, Wonju, Korea
}

\section{See Article on Page 138-143}

Treatment of rectal cancer has been improved, and the incidence of anal-sphincter-preservation surgery has increased since the popularization of a total mesorectal excision (TME) with pelvic autonomic nerve preservation [1]. Sphincter-preservation surgery with restoration of bowel continuity to avoid a permanent stoma is feasible in $50 \%-80 \%$ of patients. A preserved function after rectal surgery is directly related to better quality of life (QoL), and functional outcome has become an important parameter for defining surgical performance after a low anterior resection (LAR) $[1,2]$. However, that up to $90 \%$ of such patients will subsequently have a change in bowel habit, including fecal incontinence (FI), increased bowel frequency and emptying difficulties, what is called anterior resection syndrome (ARS), is widely accepted [3].

Currently, no specific options for treating ARS or FI exist. Pelvic floor rehabilitation (PFR), including pelvic floor muscle training (PFMT), biofeedback (BF) training, and rectal balloon training has been accepted as a standard technique for the treatment of FI [4]. A systematic review demonstrated that the use of pelvic floor training (PFT) was useful for improving functional outcome after a LAR. Most included studies showed an improvement regarding continence, stool frequency and QoL. However, the available data from studies of limited quality could not be pooled due to heterogeneity of the PFT protocols used and the different FI scoring systems [4].

In studies done in 2012, the Cochrane collaboration found some evidence that $\mathrm{BF}$ and $\mathrm{ES}$ together enhanced the outcome

Correspondence to: Ik Yong Kim, M.D.

Department of Surgery, Yonsei University Wonju College of Medicine, 20 Ilsan-ro, Wonju 26426, Korea

Tel: +82-33-741-0573, Fax: +82-33-744-6604

E-mail: iykim@yonsei.ac.kr

(C) 2015 The Korean Society of Coloproctology

This is an open-access article distributed under the terms of the Creative Commons Attribution NonCommercial License (http://creativecommons.org/licenses/by-nc/3.0) which permits unrestricted noncommercial use, distribution, and reproduction in any medium, provided the original work is properly cited. of treatment compared to ES alone or BF alone [5]. However, they concluded that the role of anal sphincter exercises and $\mathrm{BF}$ in the management of FI was not definitive; they used poor methodology and were frequently underpowered. Recently, Visser et al. [4] systematically reviewed the use of PFR after a LAR in 286 patients in five different studies and found that PFT, including BF therapy, was useful for improving functional outcome. Among the studies, only the data of Kim et al. [6] and Pucciani et al. [7] could be pooled and demonstrated improved functional outcome based on anorectal pressures and rectal capacity after the LAR. This was particularly true for patients with FI as the primary symptom [5]. Kim et al. [8] presented a timely paper on this issue in the Annals of Coloproctology. The authors evaluated the efficacy of BF in terms of the change in the defecation pattern and the QoL in patients after rectal-cancer surgery. However, major inherent limitations, such as a very small sample size in both groups, especially the treated patients (biofeedback group), still exist.

Regarding the timing of $\mathrm{BF}$ for patients with a diverting ileostomy after a LAR, BF is generally introduced after stoma takedown. In this study, the authors introduced BF before stoma closure, and they assessed the response to treatment by using anorectal manometry after stoma closure. These results have led some surgeons to be concerned about whether BF was actually required at relatively early times before stoma takedown after restoration surgery; another concern was wound healing for the neorectum.

Regarding physiology if there are no any luminal flow and no bowel peristaltic movement of the neorectum during squeezing (anorectal condition may be different or the real sampling reflex or sensory mechanism could not be tested during stoma), how visual or auditory display or instrument-assisted BF training could be used is not known. Such a situation is not adequate for assessing the objective efficacy of BF therapy for patients with ARS. Potential contributing factors, either treatment or patient related, to and the duration of PFT as a proper treatment for ARS are still under many debate [9].

As the authors' results have shown, BF therapy does not yet seem to be desirable. Still, solid evidence for the routine use of 
PFR to improve functional outcome after a LAR is lacking. So far, various types of questionnaire surveys have been used in many studies, but standardized and validated evaluation tools are lacking [10]. Nevertheless, this is a meaningful study in establishing the efficacy of BF in the management of FI after sphincter-preservation surgery. In the near future, well-designed studies using uniformly-adopted scoring systems and proper selection of sphincter-preservation-surgery patients based upon reproducible criteria are needed and may be worthwhile.

\section{CONFLICT OF INTEREST}

No potential conflict of interest relevant to this article was reported.

\section{REFERENCES}

1. Kim NK, Min BS, Kim JS, Hur H, Lee KY, Sohn SK, et al. Oncologic outcomes and safety after tumor-specific mesorectal excision for resectable rectal cancer: a single institution's experience with 1,276 patients with rectal cancer. J Korean Soc Coloproctol 2008;24:121-33.

2. Won SL, Kim IY, Sung SH, Kim DS. Functional and oncologic outcome of coloanal anastomosis in low lying rectal cancer. J Korean Soc Coloproctol 2005;21:419-25.

3. Bryant CL, Lunniss PJ, Knowles CH, Thaha MA, Chan CL. Ante- rior resection syndrome. Lancet Oncol 2012;13:e403-8.

4. Visser WS, Te Riele WW, Boerma D, van Ramshorst B, van Westreenen HL. Pelvic floor rehabilitation to improve functional outcome after a low anterior resection: a systematic review. Ann Coloproctol 2014;30:109-14.

5. Norton C, Cody JD. Biofeedback and/or sphincter exercises for the treatment of faecal incontinence in adults. Cochrane Database Syst Rev 2012;7:CD002111.

6. Kim KH, Yu CS, Yoon YS, Yoon SN, Lim SB, Kim JC. Effectiveness of biofeedback therapy in the treatment of anterior resection syndrome after rectal cancer surgery. Dis Colon Rectum 2011;54: 1107-13.

7. Pucciani F, Ringressi MN, Redditi S, Masi A, Giani I. Rehabilitation of fecal incontinence after sphincter-saving surgery for rectal cancer: encouraging results. Dis Colon Rectum 2008;51:1552-8.

8. Kim JK, Jeon BG, Song YS, Seo MS, Kwon YH, Park JW, et al. Biofeedback therapy before ileostomy closure in patients undergoing sphincter-saving surgery for rectal cancer: a pilot study. Ann Coloproctol 2015;31:138-43.

9. Byrne CM, Solomon MJ, Young JM, Rex J, Merlino CL. Biofeedback for fecal incontinence: short-term outcomes of 513 consecutive patients and predictors of successful treatment. Dis Colon Rectum 2007;50:417-27.

10. Enck P, Van der Voort IR, Klosterhalfen S. Biofeedback therapy in fecal incontinence and constipation. Neurogastroenterol Motil 2009;21:1133-41. 